\title{
Parameter-dependent Lyapunov Functions and The Discrete-time Popov Criterion for Robust
}

\section{Analysis* +}

\author{
WASSIM M. HADDAD $\ddagger$ and DENNIS S. BERNSTEIN§
}

Key Words-Parameter-dependent Lyapunov functions; constant real parameter uncertainty; robust stability and performance.

\begin{abstract}
Parameter-dependent Lyapunov functions are developed for discrete-time systems with constant real parameter uncertainty. This construction of a family of Lyapunov functions is then used to analyze robust stability with $\mathrm{H}_{2}$ performance bounds for state space systems. A special case of these results generalizes the classical discrete-time Popov criterion.

\begin{tabular}{|c|c|}
\hline $\begin{array}{l}\text { Notation } \\
\mathbb{R}, \mathbb{R}^{r \times s}, \mathbb{R}^{r} \\
\mathbb{C}, \mathbb{C}^{r \times s}, \mathbb{C}^{r}\end{array}$ & $\begin{array}{l}\text { real numbers, } r \times s \text { real matrices, } \mathbb{R}^{r \times 1} \\
\text { complex numbers, } r \times s \text { complex mat } \\
\text { rices, } \mathbb{C}^{r \times 1}\end{array}$ \\
\hline $\begin{array}{l}\mathbb{E}, \mathrm{tr}, 0_{r \times s} \\
I_{r},()^{\mathrm{T}},()^{*}\end{array}$ & $\begin{array}{l}\text { expectation, trace, } r \times s \text { zero matrix } \\
r \times r \text { identity, transpose, complex conjug } \\
\text { ate transpose } \\
\text { spectral radius, largest singular yalue }\end{array}$ \\
\hline & $\begin{array}{l}r \times r \quad \text { symmetric, negative-definite } \\
\text { positive-definite matrices }\end{array}$ \\
\hline $\begin{array}{l}Z_{1} \leq Z_{2}, Z_{1}<Z_{2} \\
\|x\|_{2} \\
\|Z\|_{F} \\
\|H(z)\|_{2}\end{array}$ & $\begin{array}{l}Z_{2}-Z_{1} \in \mathbb{N}^{r}, Z_{2}-Z_{1} \in P, Z_{1}, Z_{2} \in \mathcal{S}^{r} \\
{\left[x^{*} x\right]^{1 / 2}, x \in \mathbb{C}^{r}} \\
{\left[\operatorname{tr} Z Z^{*}\right]^{1 / 2} \text { (Frobenius matrix norm) }} \\
{\left[(1 / 2 \pi) \int_{-\pi}^{\pi}\left\|H\left(\mathrm{e}^{i \theta}\right)\right\|_{\mathrm{F}}^{2} \mathrm{~d} \theta\right]^{1 / 2}}\end{array}$ \\
\hline
\end{tabular}
\end{abstract}

\section{Introduction}

SINCE MOST physical processes evolved naturally in continuous time, it is not surprising that the bulk of robust control theory has been developed for continuous-time systems. Nevertheless, it is the overwhelming trend to implement controllers digitally. The analysis and synthesis of robust digital controllers thus requires an understanding of how the continuous-time uncertainties transform into an uncertain discrete-time model. A glimpse of the nature of this type of difficulty can be seen in Bernstein and Hollot (1989).

\footnotetext{
* Received 13 March 1992; revised 2 April 1993; received in final form $30 \mathrm{July} 1993$. This paper was not presented at any IFAC meeting. This paper was recommended for publication in revised form by Associate Editor $\mathrm{H}$. Kimura under the direction of Editor Huibert Kwakernaak. Corresponding author Professor D. S. Bernstein. Tel. +1313 764 3719; Fax +1 313763 0578; E-mail dsbaero@ engin.umich.edu.

t This research was supported in part by the Air Force Office of Scientific Research under grant F49620-92-J-0127, the National Science Foundation under Research Initiation Grant ECS-9109558 and the National Aeronautics and Space Administration under contract NAS8-38575.

¥ Department of Aerospace Engineering, Georgia Institute of Technology, Atlanta, GA 30332, U.S.A.

8 Department of Aerospace Engineering. The University of Michigan, Ann Arbor, MI 48109-2118, U.S.A.
}

The above difficulties notwithstanding, one would expect the construction of a naive (that is, not sampled-data) discrete-time robustness theory to represent a simpler task than the development of its continuous-time counterpart. Some reasons for this view include the natural limited bandwidth of discrete-time processes as well as such technicalities as the fact that, in contrast to the continuous-time case, the $\mathrm{H}_{2}$ norm of a nonstrictly proper discrete-time transfer function is finite. Experience has shown, however, that the development of discrete-time robustness theory is often an intricate affair as can be seen by the extensive activity being devoted to the development of discrete-time state space $\mathrm{H}_{\infty}$ theory.

Many practical applications of robust feedback control involve constant real parameter uncertainty, whereas small gain or norm-bounding techniques guarantee robust stability against complex, frequency-dependent uncertainty, thus entailing undue conservatism. Since conventional Lyapunov bounding techniques guarantee stability with respect to time-varying perturbations, they possess a similar drawback. This is simply due to the fact that time-varying parameter variations can destabilize a system even when the parameter variations are confined to a region in which constant variations are nondestabilizing. Consequently, a feedback controller designed for time-varying parameter variations will unnecessarily sacrifice performance when the uncertain real parameters are actually constant.

In a recent paper (Haddad and Bernstein, 1991b) a framework for parameter-dependent Lyapunov functions, a less conservative refinement of 'fixed' Lyapunov functions, was developed for continuous-time systems. It was shown by Haddad and Bernstein (1991b) that this framework provides a reinterpretation of the classical Popov criterion as a parameter-dependent Lyapunov function for constant linear parameter uncertainty.

The main contribution of the present paper is to provide a general framework for discrete-time parameter-dependent Lyapunov functions and recognize the fact that the classical discrete-time Popov criterion (Szego, 1963; Szego and Pearson, 1964; Jury and Lee, 1964a; Pearson and Gibson, 1964; Siljak and Sun, 1971) is based on a parameterdependent Lyapunov function. As a result, we obtain a generalized multivariable version of the Popov criterion for matrix-valued uncertainty (the classical Popov criterion is limited to scalar nonlinearities).

\section{Robust stability and performance problems}

Let $\mathscr{U} \subset \mathbb{R}^{n \times n}$ denote a set of perturbations $\Delta A$ of a given nominal dynamics matrix $A \in \mathbb{R}^{n \times n}$. We begin by considering the question of whether or not $A+\Delta A$ is asymptotically stable for all $\Delta A \in \mathcal{U}$.

2.1. Robust stability problem. Determine whether the linear system

$$
x(k+1)=(A+\Delta A) x(k), \quad k=0,1,2, \ldots,
$$

is asymptotically stable for all $\Delta A \in \mathcal{Q}$. 
To consider the problem of robust performance, we introduce an external white noise disturbance as in standard LQG $\left(\mathrm{H}_{2}\right)$ theory. The robust performance problem concerns the worst-case $\mathrm{H}_{2}$ norm, that is, the worst-case over $\boldsymbol{U}$ of the expected value of a quadratic form involving outputs $z(k)=E x(k)$, where $E \in \mathbb{R}^{q \times n}$, when the system is subjected to a standard white noise disturbance $w(k) \in \mathbb{R}^{d}$ with weighting $D \in \mathbb{R}^{n \times d}$.

2.2. Robust performance problem. For the disturbed linear system

$$
\begin{gathered}
x(k+1)=(A+\Delta A) x(k)+D w(k), \quad k=0,1,2, \ldots, \\
z(k)=E x(k),
\end{gathered}
$$

where $w(\cdot)$ is a zero-mean $d$-dimensional discrete-time white noise signal with covariance $I_{d}$, determine a performance bound $\beta$ satisfying

$$
J(\mathcal{q}) \triangleq \sup _{\Delta A \in \mathcal{Q}} \limsup _{k \rightarrow \infty} \mathbb{E}\left\{\|z(k)\|_{2}^{2}\right\} \leq \beta .
$$

Next, we express the $\mathrm{H}_{2}$ performance measure (4) in terms of the observability Gramian for the pair $(A+\Delta A, E)$. For convenience define $R \triangleq E^{\mathrm{T}} E, V \triangleq D D^{\mathrm{T}}$.

Lemma 2.1. Suppose $A+\Delta A$ is asymptotically stable for all $\Delta A \in q$. Then

$$
J(\mathscr{q})=\sup _{\Delta A \in q} \operatorname{tr} P_{\Delta A} V=\sup _{\Delta A \in \mathcal{Q}}\left\|G_{\Delta A}(z)\right\|_{2}^{2},
$$

where $P_{\Delta A} \in \mathbb{R}^{n \times n}$ is the unique, nonnegative-definite solution to

$$
P_{\Delta A}=(A+\Delta A)^{\mathrm{T}} P_{\Delta A}(A+\Delta A)+R,
$$

and

$$
G_{\Delta A}(z) \triangleq E[z I-(A+\Delta A)]^{-1} D .
$$

Proof. See Haddad et al. (1991).

\section{Robust stability and performance via parameter-dependent} Lyapunov functions

The key step in obtaining robust stability and performance is to bound the uncertain terms $\triangle A^{\mathrm{T}} P_{\Delta A} A+A^{\mathrm{T}} P_{\Delta A} \Delta A+$ $\Delta A^{\mathrm{T}} P \Delta A$ in the Lyapunov equation (6) by means of a parameter-dependent bounding function $\Omega(P, \Delta A)$ which guarantees robust stability by means of a family of Lyapunov functions. This corresponds to the construction of a parameter-dependent Lyapunov function. As discussed by Haddad and Bernstein (1992), this approach constrains the class of allowable time-varying uncertainties. The following result is fundamental and forms the basis for all later developments.

Theorem 3.1. Let $\Omega_{0}: \mathcal{N} \subseteq \mathbb{N}^{n} \rightarrow \mathbb{S}^{n}$ and $P_{0}: q \rightarrow \mathbb{S}^{n}$ be such that

$$
\begin{aligned}
& \Delta A^{\mathrm{T}} P A+A^{\mathrm{T}} P \Delta A+\Delta A^{\mathrm{T}} P \Delta A \leq \Omega_{0}(P) \\
&-\left[(A+\Delta A)^{\mathrm{T}} P_{0}(\Delta A)(A+\Delta A)\right. \\
&\left.-P_{0}(\Delta A)\right], \quad \Delta A \in \mathcal{Q}, \quad P \in \mathcal{N},
\end{aligned}
$$

and suppose there exists $P \in \mathcal{N}$ satisfying

$$
P=A^{\mathrm{T}} P A+\Omega_{0}(P)+R
$$

and such that $P+P_{0}(\Delta A)$ is nonnegative-definite for all $\Delta A \in q$. Then

$$
(A+\Delta A, E) \text { is detectable, } \Delta A \in \mathscr{U},
$$

if and only if

$$
A+\Delta A \text { is asymptotically stable, } \Delta A \in q .
$$

In this case,

$$
P_{\Delta A} \leq P+P_{0}(\Delta A), \quad \Delta A \in Q,
$$

where $P_{\Delta A}$ is given by (6). Therefore,

$$
J(\mathcal{U}) \leq \operatorname{tr} P V+\sup _{\Delta A \in \mathcal{Q}} \operatorname{tr} P_{0}(\Delta A) V .
$$

If, in addition, there exists $\bar{P}_{0} \in \mathbb{S}^{n}$ such that

$$
P_{0}(\Delta A) \leq \bar{P}_{0}, \quad \Delta A \in q,
$$

then

$$
J(\mathscr{U}) \leq \beta,
$$

where

$$
\beta \stackrel{\Delta}{\#}\left[\left(P+\vec{P}_{0}\right) V\right] .
$$

Proof. We stress that in (7), $P$ denotes an arbitrary element of $\mathcal{N}$, whereas in (8) $P$ denotes a specific solution of the modified Lyapunov equation (8). This minor abuse of notation considerably simplifies the presentation. Now note that, for all $\Delta A \in \mathbb{R}^{n \times n},(8)$ is equivalent to

$$
\begin{aligned}
P= & (A+\Delta A)^{\mathrm{T}} P(A+\Delta A)+\Omega_{0}(P) \\
& -\left(\Delta A^{\mathrm{T}} P A+A^{\mathrm{T}} P \Delta A+\Delta A^{\mathrm{T}} P \Delta A\right)+R .
\end{aligned}
$$

Adding and subtracting

$$
(A+\Delta A)^{\mathrm{T}} P_{0}(\Delta A)(A+\Delta A)+P_{0}(\Delta A)
$$

to and from (16) yields

$$
\begin{aligned}
P+P_{0}(\Delta A)= & (A+\Delta A)^{\mathrm{T}}\left(P+P_{0}(\Delta A)\right)(A+\Delta A)+\Omega_{0}(P) \\
& -\left[(A+\Delta A)^{\mathrm{T}} P_{0}(\Delta A)(A+\Delta A)-P_{0}(\Delta A)\right] \\
& -\left(\Delta A^{\mathrm{T}} P A+A^{\mathrm{T}} P \Delta A+\Delta A^{\mathrm{T}} P \Delta A\right)+R .
\end{aligned}
$$

Hence, by assumption, (17) has a solution $P \in \mathcal{N}$ for all $\Delta A \in \mathbb{R}^{n \times n}$. If $\Delta A$ is restricted to the set $\mathcal{Q}$ then, by (7), the expression

$$
\begin{aligned}
\mathrm{\Omega}_{0}(P)-\left[(A+\Delta A)^{\mathrm{T}} P_{0}(\Delta A)(A+\Delta A)-\right. & \left.P_{0}(\Delta A)\right] \\
& -\left(\Delta A^{\mathrm{T}} P A+A^{\mathrm{T}} P \Delta A+\Delta A^{\mathrm{T}} P \Delta A\right)
\end{aligned}
$$

is nonnegative definite. Thus, if the detectability condition (9) holds for all $\triangle A \in \mathcal{Q}$, then it follows from Theorem 3.6 of Wonham (1979) that $\left(A+\Delta A,\left[R+\Omega(P, \Delta A)-\left(\Delta A^{\mathrm{T}} P A+\right.\right.\right.$ $\left.\left.\left.A^{\mathrm{T} P \Delta A}+\Delta A^{\mathrm{T}} P \Delta A\right)\right]^{1 / 2}\right)$ is detectable for all $\Delta A \in \mathcal{U}$, where $\boldsymbol{\Omega}(\boldsymbol{P}, \Delta \boldsymbol{A})$

$$
\triangleq \Omega_{0}(P)-\left[(A+\Delta A)^{\mathrm{T}} P_{0}(\Delta A)(A+\Delta A)-P_{0}(\Delta A)\right] .
$$

It now follows from (17) and Lemma 12.2', p. 282 of Wonham (1979) that $A+\Delta A$ is asymptotically stable for all $\Delta A \in q$. Conversely, if $A+\Delta A$ is asymptotically stable for all $\triangle A \in \mathcal{U}$, then (9) is immediate. Now, subtracting (6) from (17) yields

$$
\begin{aligned}
P+ & P_{0}(\Delta A)-P_{\Delta A} \\
= & (A+\Delta A)^{\mathrm{T}}\left(P+P_{0}(\Delta A)-P_{\Delta A}\right)(A+\Delta A)+\Omega(P, \Delta A) \\
& -\left(\Delta A^{\mathrm{T}} P A+A^{\mathrm{T}} P \Delta A+\Delta A^{\mathrm{T}} P \Delta A\right), \quad \Delta A \in \mathcal{Q},
\end{aligned}
$$

or, equivalently, since $A+\Delta A$ is asymptotically stable for all $\Delta A \in \mathcal{Q}$,

$$
\begin{aligned}
P+ & P_{0}(\Delta A)-P_{\Delta A} \\
= & \sum_{i=0}^{\infty}(A+\Delta A)^{i \mathrm{~T}}\left[\Omega(P, \Delta A)-\left(\Delta A^{\mathrm{T}} P A+A^{\mathrm{T}} P \Delta A\right.\right. \\
& \left.\left.+\Delta A^{\mathrm{T}} P \Delta A\right)\right](A+\Delta A)^{i} \geq 0, \quad \Delta A \in \mathcal{U},
\end{aligned}
$$

which implies (11). The performance bounds (12) and (14) are now immediate consequences of $(5),(11)$, and (13).

Note that with $\Omega(P, \Delta A)$ defined by (18), condition (7) can be written as

$$
\begin{aligned}
\Delta A^{\mathrm{T}} P A+A^{\mathrm{T}} P \Delta A+ & \Delta A^{\mathrm{T}} P \Delta A \\
& \leq \Omega(P, \Delta A), \quad \Delta A \in \mathcal{U}, \quad P \in \mathcal{N},
\end{aligned}
$$

where $\Omega(P, \Delta A)$ is a function of the uncertain parameters $\Delta A$. For convenience we shall say that $\Omega(\cdot, \cdot)$ is a parameter-dependent bounding function or, to be consistent with Bernstein and Haddad (1990) a parameter-dependent Q-bound. One can recover the standard guaranteed cost bound or parameter-independent $\Omega$-bound as developed in Haddad et al. (1993) by setting $P_{0}(\Delta A) \equiv 0$ so that 
$\Omega(P, \Delta A)=\Omega_{0}(P)$ and therefore $\triangle A^{\mathrm{T}} P A+A^{\mathrm{T}} P \Delta A+$ $\Delta A^{\mathrm{T}} P \Delta A \leq \Omega_{0}(P)$ for all $\Delta A \in \mathcal{Q}$. Finally, since we do not assume that $P_{0}(0)=0, \Omega_{0}(P)$ need not be nonnegativedefinite. If, however, $P_{0}(0)=0$, then $\Omega_{0}(P) \geqslant 0$ for all nonnegative-definite $P$. As in the continuous-time case (Haddad and Bernstein, 1991b, 1994), to apply Theorem 3.1 we first specify a function $\Omega_{0}(\cdot)$ and an uncertainty set $q$ such that (7)' holds. If the existence of a nonnegativedefinite solution $P$ to (8) can be determined analytically or numerically and the detectability condition (9) is satisfied, then robust stability is guaranteed and the performance bound (15) can be computed.

Finally, we note that the parameter-dependent $\Omega$-bound framework establishing robust stability given by Theorem 3.1 is equivalent to the existence of a parameter-dependent Lyapunov function of the form $V(x)=x^{\mathrm{T}}\left(P+P_{0}(\Delta A)\right) x$ which also establishes robust stability. For further details see Haddad and Bernstein (1992)

4. Construction of parameter-dependent Lyapunov functions: quadratic bound and connections to the discretetime Popov criterion

Having established the theoretical basis for our approach, we now assign explicit structure to the set $\vartheta$ and the parameter-dependent bounding function $\Omega(\cdot, \cdot)$. Specifically, the uncertainty set $\mathcal{U}$ is defined by

$$
\mathscr{U} \triangleq\left\{\Delta A \in \mathbb{R}^{n \times n}: \Delta A=B_{0} F C_{0}, \text { where } F \in \mathscr{F}\right\},
$$

where $F$ is a given set of matrices satisfying

$$
\mathscr{F} \subseteq \hat{\mathscr{F}} \triangleq\left\{F \in \mathbb{R}^{m_{0} \times m_{0}}: F^{\mathrm{T}}\left(M^{-1}+M^{-\mathrm{T}}\right) F \leq F+F^{\mathrm{T}}\right\} \text {, }
$$

and where $B_{0} \in \mathbb{R}^{n \times m_{0}}, C_{0} \in \mathbb{R}^{m_{0} \times n}$ are fixed matrices denoting the structure of the uncertainty, $F \in \mathbb{R}^{m_{0} \times m_{10}}$ is an uncertain matrix, and $M$ is a given $m_{0} \times m_{0}$ invertible matrix. Note we do not exclude the possibility that $\mathscr{F}$ is equal to $\hat{F}$. However, for flexibility $\mathscr{F}$ may be a specified proper subset of $\dot{P}$.

Next, we digress slightly to provide simplified characterizations of the set $\mathscr{F}$. Define the subset $\hat{F}_{0}$ of $\hat{F}$ by

$$
\hat{\hat{F}_{0}}=\left\{\boldsymbol{F} \in \hat{F}: \operatorname{det}\left(I-M^{-1} F\right) \neq 0\right\} .
$$

Proposition 4.1. The set $\hat{\mathscr{F}}_{0}$ is equivalently characterized by $\hat{F}_{0}=\left\{F \in \mathbb{R}^{m_{0} \times m_{0}} ; F=\left(I+\hat{F} M^{-1}\right)^{-1} \hat{F}\right.$, where

$$
\left.\hat{F} \in \mathbb{R}^{m_{0} \times m_{0}}, \hat{F}+\hat{F}^{\mathrm{T}} \geq 0 \text {, and } \operatorname{det}\left(I+\hat{F} M^{-1}\right) \neq 0\right\} .
$$

Proof. See Haddad and Bernstein (1991b, 1994).

In the special case that $M$ is positive definite, it follows from Lemma 4.1 of Haddad and Bernstein (1991a, 1993b) that the condition $\operatorname{det}\left(I+\hat{F} M^{-1}\right) \neq 0$ in the definition of $\hat{F}_{0}$ is automatically satisfied. In this case we have the following norm bound inequality on $F \in \hat{F}$.

Lemma 4.1. Let $F \in \hat{F}$ and assume $M$ is positive definite. Then

$$
\sigma_{\max }(F) \leq \sigma_{\max }(M) .
$$

Proof. See Haddad and Bernstein (1994).

Next, we provide further simplification of the set $\hat{F}$ for the case in which $F$ is symmetric and $M$ is positive definite.

Lemma 4.2. Let $F \in \mathbb{S}^{m_{0}}$ and $M \in \mathbb{P}^{m_{0}}$. Then $F M^{-1} F \leq F$ if and only if $0 \leq F \leq M$.

Proof. See Haddad and Bernstein (1991b, 1994).

Thus, in the case in which $F$ is symmetric and $M$ is positive definite the set $F$ defined, by (22) becomes

$$
\hat{\mathscr{F}}_{\mathrm{S}} \triangleq\left\{F \in \mathbb{S}^{m_{0}}: 0 \leq F \leq M\right\} \text {. }
$$

Note that if $F$ in $F$ is constrained to have the diagonal structure $\operatorname{diag}\left[F_{1}, F_{2}, \ldots, F_{m_{0}}\right]$, then $0 \leq F_{i} \leq M_{i}, \quad i=$ $1, \ldots, m_{0}$, where $M=\operatorname{diag}\left[M_{1}, M_{2}, \ldots, M_{m_{0}}\right]$. More generally, $F$ may have repeated elements and/or blocks on the diagonal of the form diag $\left[F_{1}, F_{1}, F_{1}, F_{2}, \ldots, F_{m_{0}}\right]$. Finally, in accordance with our assumption in Section 2 that $0 \in \mathcal{U}$, we shall assume that $0 \in \mathbb{F}$. Also for convenience we always assume $M \in \mathscr{F}$

For the structure of $q$ satisfying (21), the parameterdependent bound $\Omega(\cdot, \cdot)$ satisfying (18) can now be given a concrete form. However, since the elements $\Delta A$ in $\ell$ are parameterized by the elements $F$ in $\mathscr{F}$, for convenience in the following results we shall write $P_{0}(F)$ in place of $P_{0}(\Delta A)$ We now introduce a key definition that will be used in subsequent developments.

Definition 4.1. Let $N \in \mathbb{R}^{m_{0} \times m_{0}}$. Then $F$ and $N$ are compatible if $F^{\mathrm{T}} N$ is symmetric for all $F \in F$. Furthermore, $F$ and $N$ are strongly compatible if, in addition, $F^{\mathrm{T}} N$ is nonnegative-definite for all $F \in \mathscr{F}$.

For the remainder of this paper we assume for simplicity that $M$ is positive definite. In this case it follows from Lemma 4.1 that there exists $\mu \in \mathbb{S}^{m_{0}}$ such that $F^{\mathrm{T}} N \leq \mu$ for all $F \in \mathscr{F}$

Proposition 4.2. Let $N \in \mathbb{R}^{m_{0} \times m_{0}}$ be such that $F$ and $N$ are compatible, and let $\mathcal{N} \subseteq \mathbb{N}^{n}$ denote the set of $P \in \mathbb{N}^{n}$ such that

Then the function

$$
\begin{aligned}
\mathcal{N} \triangleq & \left\{P \in \mathbb{N}^{n}: M^{-1}-N C_{0} B_{0}+\left(M^{-1}-N C_{0} B_{0}\right)^{\mathrm{T}}\right. \\
& \left.-B_{0}^{\mathrm{T}} C_{0}^{\mathrm{T}} \mu C_{0} B_{0}-B_{0}^{\mathrm{T}} P B_{0}>0\right\} .
\end{aligned}
$$

$$
\begin{aligned}
\Omega_{0}(P)= & (A-I)^{\mathrm{T}} C_{0}^{\mathrm{T}} \mu C_{0}(A-I) \\
& +\left(C_{0}+B_{0}^{\mathrm{T}} C_{0}^{\mathrm{T}} \mu C_{0} A+N C_{0} A\right. \\
& \left.-B_{0}^{\mathrm{T}} C^{\mathrm{T}} \mu C_{0}-N C_{0}+B_{0}^{\mathrm{T}} P A\right)^{\mathrm{T}} \\
& \cdot\left[\left(M^{-1}-N C_{0} B_{0}\right)+\left(M^{-1}-N C_{0} B_{0}\right)^{\mathrm{T}}\right. \\
& \left.-B_{0}^{\mathrm{T}} C_{0}^{\mathrm{T}} \mu C_{0} B_{0}-B_{0}^{\mathrm{T}} P B_{0}\right]^{-1} \\
& \cdot\left(C_{0}+B_{0}^{\mathrm{T}} C_{0}^{\mathrm{T}} \mu C_{0} A+N C_{0} A\right. \\
& \left.-B_{0}^{\mathrm{T}} C^{\mathrm{T}} \mu C_{0}-N C_{0}+B_{0}^{\mathrm{T}} P A\right), \\
& P_{0}(F)=C_{0}^{\mathrm{T}} F^{\mathrm{T}} N C_{0},
\end{aligned}
$$

satisfy (7) with $Q$ given by (21).

Proof. Since by (25) $M^{-1}-N C_{0} B_{0}+\left(M^{-1}-N C_{0} B_{0}\right)^{\mathrm{T}}-$ $B_{0}^{T} C_{0}^{T} \mu C_{0} B_{0}-B_{0}^{T} P B_{0}$ is positive-definite for all $P \in \mathcal{N}$ and by (22), $F+F^{\mathrm{T}}-2 F^{\mathrm{T}} M^{-1} F$ is nonnegative-definite, it follows that

$$
\begin{aligned}
0 \leq & {\left[\left(C_{0}+B_{0}^{\mathrm{T}} C_{0}^{\mathrm{T}} \mu C_{0} A+N C_{0} A-B_{0}^{\mathrm{T}} C_{0}^{\mathrm{T}} \mu C_{0}-N C_{0}+B_{0}^{\mathrm{T}} P A\right)\right.} \\
& -\left[\left(M^{-1}-N C_{0} B_{0}\right)+\left(M^{-1}-N C_{0} B_{0}\right)^{\mathrm{T}}\right. \\
& \left.\left.-B_{0}^{\mathrm{T}} C_{0}^{\mathrm{T}} \mu C_{0} B_{0}-B_{0}^{\mathrm{T}} P B_{0}\right] F C_{0}\right]^{\mathrm{T}} \\
& \cdot\left[\left(M^{-1}-N C_{0} B_{0}\right)+\left(M^{-1}-N C_{0} B_{0}\right)^{\mathrm{T}}\right. \\
& \left.-B_{0}^{\mathrm{T}} C_{0}^{\mathrm{T}} \mu C_{0} B_{0}-B_{0}^{\mathrm{T}} P B_{0}\right]^{-1} \\
& -\left[\left(C_{0}+B_{0}^{\mathrm{T}} C_{0}^{\mathrm{T}} \mu C_{0} A+N C_{0} A\right.\right. \\
& \left.-B_{0} C_{0}^{\mathrm{T}} \mu C_{0}-N C_{0}+B_{0}^{\mathrm{T}} P A\right) \\
& -\left[\left(M^{-1}-N C_{0} B_{0}\right)+\left(M^{-1}-N C_{0} B_{0}\right)^{\mathrm{T}}\right. \\
& \left.\left.-B_{0}^{\mathrm{T}} C_{0}^{\mathrm{T}} \mu C_{0} B_{0}-B_{0}^{\mathrm{T}} P B_{0}\right] F C_{0}\right] \\
& +(A-I)^{\mathrm{T}} C_{0}^{\mathrm{T}} \mu C_{0}(A-I)-(A-I)^{\mathrm{T}} C_{0}^{\mathrm{T}} \mu C_{0}(A-I) \\
& +C_{0}^{\mathrm{T}}\left[F+F^{\mathrm{T}}-2 F^{\mathrm{T}} M^{-1} F\right] C_{0} \\
= & \Omega_{0}(P)-C_{0}^{\mathrm{T}} F^{\mathrm{T}}\left(C_{0}+B_{0}^{\mathrm{T}} C_{0}^{\mathrm{T}} \mu C_{0} A+N C_{0} A\right. \\
& \left.-B_{0}^{\mathrm{T}} C_{0}^{\mathrm{T}} \mu C_{0}-N C_{0}+B_{0}^{\mathrm{T}} P A\right) \\
& -\left(C_{0}+B_{0}^{\mathrm{T}} C_{0}^{\mathrm{T}} \mu C_{0} A+N C_{0} A-B_{0}^{\mathrm{T}} C_{0}^{\mathrm{T}} \mu C_{0}\right. \\
& \left.-N C_{0}+B_{0}^{\mathrm{T}} P A\right)^{\mathrm{T}} F C_{0}+C_{0}^{\mathrm{T}} F^{\mathrm{T}}\left[\left(M^{-1}-N C_{0} B_{0}\right)\right. \\
& \left.+\left(M^{-1}-N C_{0} B_{0}\right)^{\mathrm{T}}-B_{0}^{\mathrm{T}} C_{0}^{\mathrm{T}} \mu C_{0} B_{0}-B_{0}^{\mathrm{T}} P B_{0}\right] F C_{0} \\
& -(A-I)^{\mathrm{T}} C_{0}^{\mathrm{T}} \mu C_{0}(A-I)+C_{0}^{\mathrm{T}}\left[F+F^{\mathrm{T}}-2 F^{\mathrm{T}} M^{-1} F\right] C_{0} \\
= & \Omega_{0}(P)-C_{0}^{\mathrm{T}} F^{\mathrm{T}} B_{0}^{\mathrm{T}} C_{0}^{\mathrm{T}} \mu C_{0} A-C_{0}^{\mathrm{T}} F^{\mathrm{T}} N C_{0} A \\
& +C_{0}^{\mathrm{T}} F^{\mathrm{T}} B_{0}^{\mathrm{T}} C_{0}^{\mathrm{T}} \mu C_{0}+C_{0}^{\mathrm{T}} F^{\mathrm{T}} N C_{0}-C_{0}^{\mathrm{T}} F^{\mathrm{T}} B_{0}^{\mathrm{T}} P A \\
& -A^{\mathrm{T}} C_{0}^{\mathrm{T}} \mu C_{0} B_{0} F C_{0}-A^{\mathrm{T}} C_{0}^{\mathrm{T}} N^{\mathrm{T}} F C_{0} \\
& +C_{0}^{\mathrm{T}} \mu C_{0} B_{0} F C_{0}+C_{0}^{\mathrm{T}} N^{\mathrm{T}} F C_{0}-A^{\mathrm{T}} P B_{0} F C_{0} \\
& -C_{0}^{\mathrm{T}} F^{\mathrm{T}} N C_{0} B_{0} F C_{0}-C_{0}^{\mathrm{T}} F^{\mathrm{T}} B_{0}^{\mathrm{T}} C_{0}^{\mathrm{T}} N^{\mathrm{T}} F C_{0} \\
& -C_{0}^{\mathrm{T}} F^{\mathrm{T}} B_{0}^{\mathrm{T}} C_{0}^{\mathrm{T}} \mu C_{0} B_{0} F C_{0}-C_{0}^{\mathrm{T}} F^{\mathrm{T}} B_{0}^{\mathrm{T}} P B_{0} F C_{0} \\
& -(A-I) C_{0}^{\mathrm{T}} \mu C_{0}(A-I) . \\
& -(28) \\
&
\end{aligned}
$$


Next, setting $\bar{P}_{0} \triangleq C_{0}^{\mathrm{T}} \mu C_{0}$ and noting $\bar{P}_{0} \geq P_{0}(F)$, (28) becomes

$$
\begin{aligned}
0 \leq & \Omega_{0}(P)-\left[\Delta A^{\mathrm{T}} P A+A^{\mathrm{T}} P \Delta A+\Delta A^{\mathrm{T}} P \Delta A\right] \\
& -\left[(A+\Delta A)^{\mathrm{T}} \bar{P}_{0}(A+\Delta A)+\left[\bar{P}_{0}-P_{0}(F)\right]\right. \\
& -P_{0}(F)-\Delta A^{\mathrm{T}}\left[\bar{P}_{0}-P_{0}(F)\right]-\left[\bar{P}_{0}-P_{0}(F)\right] \Delta A \\
& -A^{\mathrm{T}}\left[\bar{P}_{0}-P_{0}(F)\right]-\left[\bar{P}_{0}-P_{0}(F)\right] A \\
= & \Omega_{0}(P)-\left[\Delta A^{\mathrm{T}} P A+A^{\mathrm{T}} P \Delta A+\Delta A^{\mathrm{T}} P \Delta A\right] \\
& -\left[(A+\Delta A)^{\mathrm{T}}\left[\ddot{P}_{0}-P_{0}(F)\right](A+\Delta A)\right. \\
& +\left[\bar{P}_{0}-P_{0}(F)\right]-\Delta A^{\mathrm{T}}\left[\bar{P}_{0}-P_{0}(F)\right] \\
& -\left[\bar{P}_{0}-P_{0}(F)\right] \Delta A-A^{\mathrm{T}}\left[\bar{P}_{0}-P_{0}(F)\right] \\
& \left.-\left[\bar{P}_{0}-P_{0}(F)\right] A+(A+\Delta A)^{\mathrm{T}} P_{0}(F)(A+\Delta A)-P_{0}(F)\right],
\end{aligned}
$$

or, equivalently,

$$
\begin{aligned}
0 \leq & {[(A-I)+\Delta A]^{\mathrm{T}}\left[\bar{P}_{0}-P_{0}(F)\right][(A-I)+\Delta A] \leq \Omega_{0}(P) } \\
& -\left[\Delta A^{\mathrm{T}} P A+A^{\mathrm{T}} P \Delta A+\Delta A^{\mathrm{T}} P \Delta A\right] \\
& -\left[(A+\Delta A)^{\mathrm{T}} P_{0}(F)(A+\Delta A)-P_{0}(F)\right],
\end{aligned}
$$

which proves $(7)$ with $\mathscr{Q}$ given by $(21)$.

Next, using Theorem 3.1 and Proposition 4.2 we have the following immediate result.

Theorem 4.1. Let $N \in \mathbb{R}^{m_{0} \times m_{0}}$ be such $\mathscr{F}$ and $N$ are strongly compatible. Furthermore, suppose there exists a nonnegative-definite matrix $P$ satisfying (25) and

$$
\begin{aligned}
P= & A^{\mathrm{T}} P A+(A-I)^{\mathrm{T}} C_{0}^{\mathrm{T}} \mu C_{0}(A-I) \\
& +\left(C_{0}+B_{0}^{\mathrm{T}} C_{0}^{\mathrm{T}} \mu C_{0} A+N C_{0} A\right. \\
& \left.-B_{0}^{\mathrm{T}} C_{0}^{\mathrm{T}} \mu C_{0}-N C_{0}+B_{0}^{\mathrm{T}} P A\right)^{\mathrm{T}} \\
& \cdot\left[\left(M^{-1}-N C_{0} B_{0}\right)+\left(M^{-1}-N C_{0} B_{0}\right)^{\mathrm{T}}\right. \\
& \left.-B_{0}^{\mathrm{T}} C_{0}^{\mathrm{T}} \mu C_{0} B_{0}-B_{0}^{\mathrm{T}} P B_{0}\right]^{-1} \\
& \cdot\left(C_{0}+B_{0}^{\mathrm{T}} C_{0}^{\mathrm{T}} \mu C_{0} A+N C_{0} A\right. \\
& \left.-B_{0}^{\mathrm{T}} C_{0}^{\mathrm{T}} \mu C_{0}-N C_{0}+B_{0}^{\mathrm{T}} P A\right)+R .
\end{aligned}
$$

Then

$$
(A+\Delta A, E) \text { is detectable, } \Delta A \in \mathcal{U},
$$

if and only if

$$
A+\Delta A \text { is asymptotically stable, } \Delta A \in \mathscr{q} \text {. }
$$

In this case,

$$
J(q) \leq \operatorname{tr}\left[\left(P+C_{0}^{\mathbf{T}} \mu C_{0}\right) V\right] .
$$

Proof. The result is a direct specialization of Theorem 3.1 using Proposition 4.2. We only note that $P_{0}(\Delta A)$ now has the form $P_{0}(F)=C_{0}^{\mathrm{T}} F^{\mathrm{T}} N C_{0}$. Since, by assumption, $F^{\mathrm{T}} N \geq 0$ for all $F \in \mathscr{F}$, it follows that $P+P_{0}(F)$ is nonnegative-definite for all $F \in F$ as required by Theorem 3.1. Finally, (32) is an immediate consequence of (14) with $\bar{P}_{0}=C_{0}^{\mathrm{T}} \mu C_{0}$.

Remark 4.1. The condition that $F^{\mathrm{T}} N=N^{\mathrm{T}} F, F \in \mathscr{F}$, represents an intimate relationship between the matrix $N$ and the structure of $\mathscr{F}$. It is easy to see that there always exists such a matrix $N$. For example, if $F=F_{0} I_{m_{0}}$, where $F_{0} \in \mathbb{R}$, then $N$ can be an arbitrary nonnegative-definite matrix. Alternatively, if $N=N_{0} I_{m_{0}}$, then $F$ may be nondiagonal. Of course, $F$ and $N$ may have more intricate structure, for example, they may be block diagonal with commuting blocks situated on the diagonal.

Next, we establish connections between the parameterdependent bounding function formed from (26) and the classical discrete-time Popov criterion (Szego, 1963; Tsypkin, 1964; Szego and Pearson, 1964; Pearson and Gibson, 1964; Jury and Lee, 1964b; Narendra and Cho, 1968; Siljak and Sun, 1971). Furthermore, by exploiting results from positivity theory it is possible to guarantee the existence of a positive-definite solution to (29). First, however, we present additional notation and definitions and review the discretetime positive real lemma used to characterize positive realness in the state-space setting (Hitz and Anderson, 1969).
Let

$$
G(z)^{\min }\left[\begin{array}{l|l}
A & B \\
\hline C & D
\end{array}\right]
$$

denote a state space realization of a transfer function $G(z)$, that is, $G(z)=C(z I-A)^{-1} B+D$. The notation 'min' denotes a minimal realization. Furthermore, an asymptotically stable transfer function is a transfer function each of whose poles is in the open unit disk.

A square transfer function $G(z)$ is called positive real if (Hitz and Anderson, 1969; Siljak, 1973) (1) all poles of $G(z)$ are in the closed unit disk, and (2) $G(z)+G^{*}(z)$ is nonnegative-definite for all $|z|>1$. A square transfer function $G(z)$ is called strictly positive real if (Caines, 1989) (1) $G(z)$ is asymptotically stable, and (2) $G\left(e^{j \theta}\right)+G\left(e^{j \theta}\right)$ is positive definite for all $\theta \in[0,2 \pi]$. Finally, a square transfer function $G(z)$ is strongly positive real if it is strictly positive real and $D+D^{\mathrm{T}}>0$, where $D \stackrel{\Delta}{=} G(\infty)$.

Lemma 4.3 (discrete-time positive real lemma). $G(z)^{\text {min }}$ $\left[\begin{array}{l|l}A & B \\ \hline C & D\end{array}\right]$ is positive real if and only if there exist real matrices $P, L$, and $W$ with $P$ positive-definite such that

$$
\begin{gathered}
P=A^{\mathrm{T}} P A+L^{\mathrm{T}} L, \\
0=B^{\mathrm{T}} P A-C+W^{\mathrm{T}} L, \\
0=D+D^{\mathrm{T}}-B^{\mathrm{T}} P B-W^{\mathrm{T}} W .
\end{gathered}
$$

Proof. See Hitz and Anderson (1969).

Next, suppose that $D+D^{\mathrm{T}}-B^{\mathrm{T}} P B>0$ where $P$ satisfies (33)-(35). Then, since

$$
W^{\mathrm{T}} W=D+D^{\mathrm{T}}-B^{\mathrm{T}} P B
$$

it follows that $W^{\mathrm{T}} W$ is nonsingular, and (34) implies

$$
W^{\mathrm{T}} L=-\left(B^{\mathrm{T}} P A-C\right)
$$

Using (37) and noting as in Haddad and Bernstein (1993) that $L^{\mathrm{T}} L \geq L^{\mathrm{T}} W\left(W^{\mathrm{T}} W\right)^{-1} W^{\mathrm{T}} L$, it follows from (33) that

$$
P \geq A^{\mathrm{T}} P A+\left(B^{\mathrm{T}} P A-C\right)^{\mathrm{T}}\left(W^{\top} W\right)^{-1}\left(B^{\mathrm{T}} P A-C\right),
$$

or, since $\left(W^{\mathrm{T}} W\right)^{-1}=\left(D+D^{\mathrm{T}}-B^{\mathrm{T}} P B\right)^{-1}$,

$$
\begin{aligned}
P \geq & A^{\mathrm{T}} P A+\left(B^{\mathrm{T}} P A-C\right)^{\mathrm{T}}\left(D+D^{\mathrm{T}}-B^{\mathrm{T}} P B\right)^{-1} \\
& \cdot\left(B^{\mathrm{T}} P A-C\right) .
\end{aligned}
$$

Using the Riccati inequality version of (39) to characterize positive realness, we have the following result.

Lemma 4.4. Let $G(z) \min \left[\begin{array}{l|l}A & B \\ C & D\end{array}\right]$. Then the following statements are equivalent:

(i) $A$ is asymptotically stable and $G(z)$ is strongly positive-real; and

(ii) there exist positive-definite matrices $P$ and $R$ such that

$$
\begin{gathered}
D+D^{\mathrm{T}}-B^{\mathrm{T}} P B>0, \\
P=A^{\mathrm{T}} P A+\left(B^{\mathrm{T}} P A-C\right)^{\mathrm{T}}\left(D+D^{\mathrm{T}}-B^{\mathrm{T}} P B\right)^{-1} \\
\cdot\left(B^{\mathrm{T}} P A-C\right)+R .
\end{gathered}
$$

Proof. The proof is similar to the proof of Lemma 4.1 for the continuous-time case given in Haddad and Bernstein $(1991 \mathrm{a}, \mathrm{b})$ and hence is omitted.

Next we use Lemma 4.4 to obtain a sufficient condition for the existence of a solution to (29).

Theorem 4.2. Let $\mathscr{G}(z) \triangleq M^{-1}+[I+(z-1) N] G(z)-$ $\frac{1}{2}|z-1|^{2} G^{*}(z) \mu G(z)$, where $G(z)$ min $\left[\begin{array}{c|c}A & -B_{0} \\ \hline C_{0} & 0\end{array}\right]$. If $A$ is asymptotically stable and $\mathscr{G}(z)$ is strongly positive real, then there exists an $n \times n$ matrix $P>0$ satisfying (29). Conversely, if there exists $P>0$ satisfying (25) and (29) for 
all $R>0$, then $A$ is asymptotically stable and $\mathscr{G}(z)$ is strongly positive real.

Proof. The proof is an immediate consequence of Lemma 4.4. Specifically, the existence of a positive-definite $P$ satisfying (29) follows from spectral factorization theory. The converse follows from algebraic manipulations. For a similar proof see Kalman and Szego (1963).

Traditionally, the classical discrete-time Popov criterion is stated for sector-bounded slope-restricted time-invariant memoryless nonlinearities. Next, we show that Theorem 4.1 is a generalization of the discrete-time Popov criterion, as proposed by Szego (1963), Jury and Lee (1964a) and Pearson and Gibson (1964), for the case in which the sector-bounded nonlinearity represents linear parameter uncertainty. To do this we first provide a self-contained proof of the generalization of the discrete-time multivariable Popov criterion for a diagonal nonlinearity structure. Specifically, we define the set $\Phi$ characterizing a class of sector-bounded slope-restricted time-invariant nonlinearities. Let $M \in$ $\mathbf{R}^{m_{0} \times m_{0}}$ be a given positive-definite matrix and define

$$
\begin{aligned}
\Phi \triangleq\{ & \phi: \mathbb{R}^{m_{0}} \rightarrow \mathbb{R}^{m_{0}}: \phi^{\mathrm{T}}(y)\left[M^{-1} \phi(y)-y\right] \leq 0, \\
& y \in \mathbb{R}^{m_{0}}, \phi(y)=\left[\phi_{1}\left(y_{1}\right), \phi_{2}\left(y_{2}\right), \ldots, \phi_{m}\left(y_{m}\right)\right]^{\mathrm{T}}, \\
& \text { and } \left.0<\frac{\phi_{i}\left(y_{i}(k+1)\right)-\phi_{i}\left(y_{i}(k)\right)}{y_{i}(k+1)-y_{i}(k)}<\mu_{i}, i=1, \ldots, m\right\} .
\end{aligned}
$$

In the scalar case $m_{0}=1$, the sector condition characterizing $\Phi$ is equivalent to the more familiar condition

$$
0 \leq \phi(y) y \leq M y^{2}, \quad y \in \mathbb{R} .
$$

For convenience in stating the multivariable generalization of the discrete-time Popov criterion we define $\mu \stackrel{\Delta}{=}$ diag $\left[\mu_{1}, \mu_{2}, \ldots, \mu_{m_{0}}\right]$ and $N \triangleq \operatorname{diag}\left[N_{1}, N_{2}, \ldots, N_{m_{0}}\right]$.

Theorem 4.3 (the discrete-time Popov criterion). If there exists a nonnegative-definite diagonal matrix $N$ such that $M^{-1}+[I+(z-1) N] G(z)-\frac{1}{2}|z-1|^{2} G^{*}(z) \mu N G(z)$

strongly discrete-time positive real, where $G(z)^{\operatorname{main}}\left[\begin{array}{l|l}A & B \\ \hline C & 0\end{array}\right]$, then the negative feedback interconnection of $G(z)$ and $\phi(\cdot)$ is asymptotically stable for $\phi(\cdot) \in \Phi$.

Proof. First note that the negative feedback interconnection of $G(z)$ and $\phi(\cdot)$ has the state space representation

$$
\begin{gathered}
x(k+1)=A x(k)-B \phi(y(k)), \\
y(k)=C x(k) .
\end{gathered}
$$

Next, since $(z-1) G(z)$ has a minimal realization

$$
(z-1) G(z)^{\min }\left[\begin{array}{c|c}
A & B \\
\hline C(A-I) & C B
\end{array}\right]
$$

it follows from Lemma 4.3 and Lemma 4.4 that if $M^{-1}+$ $[I+(z-1) N] G(z)-\frac{1}{2}|z-1|^{2} G^{*}(z) \mu N G(z)$ is strongly discrete-time positive real, then there exist $P, L, W$ with $P$ positive-definite and $W^{\top} W>0$ satisfying

$$
\begin{aligned}
P= & A^{\mathrm{T}} P A+L^{\mathrm{T}} L+(A-I)^{\mathrm{T}} C^{\mathrm{T}} \mu N C(A-I), \\
0= & B^{\mathrm{T}} P A-C+B^{\mathrm{T}} C^{\mathrm{T}} \mu N C A-B^{\mathrm{T}} C^{\mathrm{T}} \mu N C \\
- & N C A+N C+W^{\mathrm{T}} L, \\
0= & \left(M^{-1}+N C B\right)+\left(M^{-1}+N C B\right)^{\mathrm{T}} \\
& -B^{\mathrm{T}} C^{\mathrm{T}} \mu N C B-B^{\mathrm{T}} P B-W^{\mathrm{T}} W .
\end{aligned}
$$

Next, with $W^{\mathrm{T}} W>0$, it follows that $P>0$ satisfies (44)-(46) if and only if there exists $R>0$ such that $P$ satisfies

$$
\begin{aligned}
P= & A^{\mathrm{T}} P A+(A-I)^{\mathrm{T}} C^{\mathrm{T}} \mu N C(A-I) \\
& +\left(C+B^{\mathrm{T}} C^{\mathrm{T}} \mu N C+N C A\right. \\
& \left.-B^{\mathrm{T}} C^{\mathrm{T}} \mu N C A-N C-B^{\mathrm{T}} P A\right)^{\mathrm{T}} \\
& \cdot\left[\left(M^{-1}+N C B\right)+\left(M^{-1}+N C B\right)^{\mathrm{T}}\right. \\
& \left.-B^{\mathrm{T}} C^{\mathrm{T}} \mu N C B-B^{\mathrm{T}} P B\right]^{-1}
\end{aligned}
$$

$$
\begin{aligned}
& \cdot\left(C+B^{\mathrm{T}} C^{\mathrm{T}} \mu N C+N C A\right. \\
& \left.-B^{\mathrm{T}} C^{\mathrm{T}} \mu N C A-N C-B^{\mathrm{T}} P A\right)+R .
\end{aligned}
$$

Next, for $\phi \in \Phi$ define the Lyapunov candidate

$$
V(x)=x^{\mathrm{T}} P x+2 \sum_{i=1}^{m} \int_{0}^{y_{i}(k)} \phi_{i}(\sigma) N_{i} \mathrm{~d} \sigma .
$$

Note that since $P$ is positive-definite and $\phi \in \Phi, V(x)$ is positive-definite for all nonzero $x$. Thus, the corresponding Lyapunov difference is given by

$$
\begin{aligned}
\Delta V(x)= & x^{\mathrm{T}}\left(A^{\mathrm{T}} P A-P\right) x-x^{\mathrm{T}} A^{\mathrm{T}} P B \phi-\phi^{\mathrm{T}} B^{\mathrm{T}} P A x \\
& +\phi^{\mathrm{T}} B^{\mathrm{T}} P B \phi+2 \sum_{i=1}^{m} \int_{y_{i}(k)}^{y_{i}(k+1)} \phi_{i}(\sigma) N_{i} \mathrm{~d} \sigma .
\end{aligned}
$$

Next, using the fact that $0<\frac{\phi_{i}\left(y_{i}(k+1)\right)-\phi_{i}\left(y_{i}(k)\right)}{y_{i}(k+1)-y_{i}(k)}<\mu_{i}$, it follows from the mean value theorem that the last term in (49) is bounded from above by

$$
\begin{aligned}
2 \sum_{i=1}^{m} \int_{y_{i}(k)}^{y_{i}(k+1)} \phi_{i}(\sigma) N_{i} \mathrm{~d} \sigma \leqslant & \sum_{i=1}^{m}\left\{2 \phi _ { i } ( y _ { i } ( k ) ) N _ { i } \left[y_{i}(k+1)\right.\right. \\
& \left.\left.-y_{i}(k)\right]+\mu_{i} N_{i}\left[y_{i}(k+1)-y_{i}(k)\right]^{2}\right\}
\end{aligned}
$$

or, equivalently,

$$
\begin{aligned}
2 \sum_{i=1}^{m} \int_{y_{i}(k)}^{y_{i}(k+1)} \phi_{i}(\sigma) N_{i} \mathrm{~d} \sigma \leq 2 \phi^{\mathrm{T}}(y(k)) N[y(k+1)-y(k)] \\
+[y(k+1)-y(k)]^{\mathrm{T}} \mu N[y(k+1)-y(k)] .
\end{aligned}
$$

Next, since $y(k+1)=C x(k+1)=C A x(k)-C B \phi(y(k))$ and $y(k+1)-y(k)=C(A-I) x(k)-C B \phi(y(k))$, using (47), (49) becomes

$$
\begin{aligned}
\Delta V(x) \leq & -x^{\mathrm{T}} R x-x^{\mathrm{T}}(A-I)^{\mathrm{T}} C^{\mathrm{T}} \mu C(A-I) x-x^{\mathrm{T}} Q x \\
& +\phi^{\mathrm{T}} N C(A-I) x+x^{\mathrm{T}}(A-I)^{\mathrm{T}} C^{\mathrm{T}} N \phi-\phi^{\mathrm{T}} N C B \phi \\
& -\phi^{\mathrm{T}} B^{\mathrm{T}} C^{\mathrm{T}} N \phi+x^{\mathrm{T}}(A-I)^{\mathrm{T}} C^{\mathrm{T}} \mu N C(A-I) x \\
& -x^{\mathrm{T}}(A-I)^{\mathrm{T}} C \mu N C B \phi-\phi^{\mathrm{T}} B^{\mathrm{T}} C^{\mathrm{T}} \mu N C(A-I) x \\
& +\phi^{\mathrm{T}} B^{\mathrm{T}} C^{\mathrm{T}} \mu N C B \phi-x^{\mathrm{T}} A^{\mathrm{T}} P B \phi-\phi^{\mathrm{T}} B^{\mathrm{T}} P A x \\
& +\phi^{\mathrm{T}} B^{\mathrm{T}} P B \phi,
\end{aligned}
$$

where

$$
\begin{gathered}
Q \triangleq\left(C+B^{\mathrm{T}} C^{\mathrm{T}} \mu N C+N C A-B^{\mathrm{T}} C^{\mathrm{T}} \mu N C A-N C-B^{\mathrm{T}} P A\right)^{\mathrm{T}} \\
\cdot\left[\left(M^{-1}+N C B\right)+\left(M^{-1}+N C B\right)^{\mathrm{T}}-B^{\mathrm{T}} C^{\mathrm{T}} \mu N C B-B^{\mathrm{T}} P B\right]^{-1} \\
\cdot\left(C+B^{\mathrm{T}} C^{\mathrm{T}} \mu N C+N C A-B^{\mathrm{T}} C^{\mathrm{T}} \mu N C A-N C-B^{\mathrm{T}} P A\right) .
\end{gathered}
$$

Adding and subtracting $2 \phi^{\mathrm{T}} C x$ and $2 \phi^{\mathrm{T}} M^{-1} \phi$ to and from (50) yields

$$
\begin{aligned}
\Delta V(x) \leq & -x^{\mathrm{T}} R x-x^{\mathrm{T}} Q x+\phi^{\mathrm{T}}\left[C+B^{\mathrm{T}} C^{\mathrm{T}} \mu N C(I-A)\right. \\
& \left.+N C(A-I)-B^{\mathrm{T}} P A\right] x \\
& +x^{\mathrm{T}}\left[C+B^{\mathrm{T}} C^{\mathrm{T}} \mu N C(I-A)+N C(A-I)-B^{\mathrm{T}} P A\right]^{\mathrm{T}} \phi \\
& -\phi^{\mathrm{T}}\left[M^{-1}+N C B\right)+\left(M^{-1}+N C B\right)^{\mathrm{T}} \\
& \left.-B^{\mathrm{T}} C^{\mathrm{T}} \mu N C B-B^{\mathrm{T}} P B\right] \phi \\
& +2 \phi^{\mathrm{T}}\left[M^{-1} \phi-C x\right]
\end{aligned}
$$

or, equivalently,

$$
\Delta V(x) \leq-x^{\mathrm{T}} R x-z^{\mathrm{T}} z+2 \phi^{\mathrm{T}}\left[M^{-1} \phi-y\right],
$$

where

$$
\begin{aligned}
z \triangleq & {\left[\left(M^{-1}+N C B\right)+\left(M^{-1}+N C B\right)^{\mathrm{T}}\right.} \\
& \left.-B^{\mathrm{T}} C^{\mathrm{T}} \mu N C B-B^{\mathrm{T}} P B\right]^{-1 / 2}\left[C+B^{\mathrm{T}} C^{\mathrm{T}} \mu N C(I-A)\right. \\
& \left.+N C(A-I)-B^{\mathrm{T}} P A\right]-\left[\left(M^{-1}+N C B\right)\right. \\
& \left.+\left(M^{-1}+N C B\right)^{\mathrm{T}}-B^{\mathrm{T}} C^{\mathrm{T}} \mu N C B-B^{\mathrm{T}} P B\right]^{1 / 2} \phi .
\end{aligned}
$$

Since $R$ is positive-definite and $\phi^{\top}\left[M^{-1} \phi-y\right] \leq 0$, it follows that $\Delta V(x)$ is negative definite.

Next, we specialize the results of Theorem 4.3 to robust stability with constant linear parameter uncertainty. Specifi- 
cally, consider the system

$$
x(k+1)=(A+\Delta A) x(k),
$$

where $\Delta A \in \mathscr{U}$ and $\mathscr{U}$ is defined by

$$
\begin{gathered}
q \stackrel{\Delta}{\{}\left\{\Delta A: \Delta A=-B F C, F=\operatorname{diag}\left[F_{1}, F_{2}, \ldots, F_{m_{0}}\right],\right. \\
\left.0 \leq F_{i} \leq M_{i}, i=1, \ldots, m_{0}\right\} .
\end{gathered}
$$

It now follows from Theorem 4.3 by setting $\phi(y)=F y=F C x$ that $A+\Delta A$ is asymptotically stable for all $\Delta A \in \mathcal{U}$.

It has thus been shown that in the special case that $F$ and $N$ are diagonal nonnegative-definite matrices, Theorem 4.1 (with $B_{0}$ replaced by $-B_{0}$ ) specializes to the discrete-time Popov criterion when applied to linear parameter uncertainty. This is not surprising since the Lyapunov function (48) that establishes robust stability has the form

$$
V(x)=x^{\mathrm{T}} P x+2 \sum_{i=1}^{m_{0}} \int_{0}^{y_{i}} F_{i} \sigma N_{i} \mathrm{~d} \sigma, \quad y_{i}=\left(C_{0} x\right)_{i},
$$

or, equivalently,

$$
V(x)=x^{\mathrm{T}} P x+x^{\mathrm{T}} C_{0}^{\mathrm{T}} F N C_{0} x=x^{\mathrm{T}} P x+\sum_{i=1}^{m_{0}} F_{i} N_{i} x^{\mathrm{T}} C_{0}^{\mathrm{T}} C_{0} x
$$

and thus, is a special case of the parameter-dependent Lyapunov function discussed earlier. Note that the uncertain parameters are not allowed to be arbitrarily time-varying, which is consistent with the fact that the classical discrete-time Popov criterion is restricted to time-invariant nonlinearities.

Finally, we consider a specialization of Proposition 4.2 and Theorem 4.1 to the case in which $N=0, \mu=0$, and $2 M^{-1}=D_{0}+D_{0}^{\mathrm{T}}>0$. In this case we have the following result.

Proposition 4.3. Let $D_{n} \in \mathbb{R}^{m_{0} \times m_{0}}$, and let $\mathcal{N} \subseteq \mathbb{N}^{n}$ denote the set of $P \in \mathbb{N}^{n}$ such that

$$
\mathcal{N} \triangleq\left\{P \in \mathbb{N}^{n}: D_{0}+D_{0}^{\mathrm{T}}-B_{0}^{\mathbf{T}} P B_{0}>0\right\} .
$$

Then the functions

$$
\Omega_{0}(P)=\left(C_{0}+B_{0}^{\mathrm{T} P A}\right)^{\mathrm{T}}\left(D_{0}+D_{0}^{\mathrm{T}}-B_{0}^{\mathrm{T}} P B_{0}\right)^{-1}\left(C_{0}+B_{0}^{\mathrm{T} P A}\right),
$$

$$
P_{0}(F)=0
$$

satisfy (7) with $q$ given by (21).

Proof. The proof is an immediate consequence of Proposition 4.2 with $N=0$.

Because of (58), the case $N=0$ corresponds to a parameter-independent $\Omega$-bound. Hence, it follows from Theorem 3.1 that if there exists a nonnegative-definite matrix $P$ satisfying

$$
\begin{aligned}
P= & A^{\mathrm{T}} P A+\left(C_{0}+B_{0}^{\mathrm{T}} P A\right)^{\mathrm{T}}\left(D_{0}+D_{0}^{\mathrm{T}}-B_{0}^{\mathrm{T}} P B_{0}\right)^{-1} \\
& \cdot\left(C_{0}+B_{0}^{\mathrm{T}} P A\right)+R,
\end{aligned}
$$

then $(A+\Delta A, E)$ is detectable for all $\Delta A \in \mathscr{U}$ if and only if $A+\Delta A$ is asymptotically stable for all $\Delta A \in \mathcal{Q}$. Using the characterization for $F \in \mathbb{F}_{0}$ implies that

$$
\Delta A=B_{0} F C_{0}=B_{0}\left(I+\hat{F} D_{0}\right)^{-1} \hat{F} C_{0},
$$

where $\hat{F}+\hat{F}^{\mathrm{T}} \geq 0$.

Furthermore, it follows from Lemma 4.4 that the existence of a positive-definite matrix $P$ satisfying (59) implies that

$$
C(z) \sim\left[\begin{array}{c|c}
A & -B_{0} \\
\hline C_{0} & D_{0}
\end{array}\right]
$$

is strongly positive real. Hence the parameter-independent
$\Omega$-bound (57) guarantees robust stability in the presence of positive real (but otherwise unknown) plant uncertainty. Hence, as in discrete-time $H_{\infty}$ bounded-real theory, it can be seen that discrete-time positive real theory is based upon yet another $\Omega$-bound. This is not surprising since similar remarks apply in the continuous-time case (Haddad and Bernstein, 1993).

\section{Conclusion}

In this paper we developed a theory of discrete-time parameter-dependent Lyapunov functions. These Lyapunov functions were used to guarantee robust stability and performance for sector-bounded slope-restricted nonlinearities which include linear parametric uncertainty as a special case.

\section{References}

Bernstein, D. S. and W. M. Haddad (1990). Robust stability and performance analysis for state space systems via quadratic Lyapunov bounds. SIAM J. Matrix Anal. Appl., 11, 239-271.

Bernstein, D. S. and C. V. Hollot (1989). Robust stability for sampled-data systems. Sys. Contr. Lett. 13, 217-226.

Caines, P. W. (1989). Linear Stochastic Systems. Wiley, NY.

Haddad, W. M. and D. S. Bernstein (1991a). Robust stabilization with positive real uncertainty: beyond the small gain theorem. Syst. Contr. Lett., 17, 191-208.

Haddad, W. M. and D. S. Bernstein (1991b). Parameterdependent Lyapunov functions, constant real parameter uncertainty, and the Popov criterion in robust analysis and synthesis, part 1 and 2. Proc. IEEE Conf. Dec. Contr., 2274-2279; 2632-2633.

Haddad, W. M. and D. S. Bernstein (1992). Parameterdependent Lyapunov functions and the discrete-time Popov criterion for robust analysis and synthesis. Proc. Amer. Contr. Conf., 594-598.

Haddad, W. M. and D. S. Bernstein (1993). Explicit construction of quadratic Lyapunov function for the small gain, positivity, circle, and Popov theorems and their application to robust stability, part I; continuous-time theory; part II: discrete-time theory. Int. J. Robust and Nonlinear Control, 3, 313-339.

Haddad, W. M. and D. S. Bernstein (1994). Parameterdependent Lyapunov functions, constant real parameter uncertainty, and the Popov criterion in robust analysis and synthesis. IEEE Trans. Autom. Contr., 39, to appear.

Haddad, W. M., D. S. Bernstein and D. Mustafa (1991). Mixed-norm $\mathrm{H}_{2} / \mathrm{H}_{\infty}$ regulation and estimation: the discrete-time case. Sys. Contr. Lett., 16, 235-248.

Haddad, W. M., H.-H. Huang and D. S. Bernstein (1993). Robust stability and performance via fixed-order dynamic compensation: the discrete-time case. IEEE Trans. Autom. Control, 38, 776-782.

Hitz, L. and B. D. O. Anderson (1969). Discrete positive-real functions and their application to system stability. Proc. IEE, 116, 153-155.

Jury, E. I. and B. W. Lee (1964a). On the stability of a certain class of nonlinear sampled-data systems. IEEE Trans. Autom. Contr., AC-9, 51-61.

Jury, E.I. and B.W. Lee (1964b). On the absolute stability of nonlinear sampled-data systems. IEEE Trans. Autom. Contr., AC-9, 551-554.

Kalman, R.E. and G. Szego (1963). Sur la stabilité d'un système d'équations aux différences finies. CR Acad. Sci., 388-390.

Narendra, K. S. and Y.-S. Cho (1968). Stability analysis of nonlinear and time-varying discrete-systems. SIAM J. Contr., 6, 625-646.

Pearson, Jr., J. B. and J. E. Gibson (1964). On the asymptotic stability of a class of saturating sampled-data systems. IEEE Trans. Appl. Ind., 83, 81-86.

Siljak, D. D. (1973). Algebraic criteria for positive realness relative to the unit circle. J. Franklin Inst., 295, 469-476. 
Siljak, D. D. and C. K. Sun (1971). Exponential absolute stability of discrete systems. ZAMM, 51, 271-275.

Szego, G. (1963). On the absolute stability of sampled-data control systems. Proc. Nat'l Acad. Sci., 49, 558-560.

Szego, G. and J. B. Pearson, Jr. (1964). On the absolute stability of sampled-data systems the indirect control case.
IEEE Trans. Autom. Contr., AC-9, 160-163.

Tsypkin, Y. Z. (1964). A criterion for absolute stability of automatic pulse systems with monotonic characteristics of the nonlinear element. Sov. Phys.-Doklady, 9, 263-266.

Wonham, W. M. (1979). Linear Multivariable Control: A Geometric Approach. Springer-Verlag, NY. 